\title{
PROPAGATION OF LOW-FREQUENCY ELECTROMAGNETIC WAVES ACROSS A MULTILAYER CYLINDRICAL SHELL
}

\begin{abstract}
In the paper the results of the analysis of propagation of the low-frequency electromagnetic waves across the multilayer cylindrical shell are presented. The mathematical model of non-local bilateral boundary conditions of multilayer cylindrical shells was created. These non-local bilateral boundary conditions describe the penetration of low-frequency electromagnetic waves across layers of the shell. The method of transformation of the boundary problem to the integral Fredholm equations of the second kind was developed. Furthermore, the value of efficiency coefficient of the shell was calculated. Analytical transformations are based on the Bessel functions.
\end{abstract}

Keywords: Bessel function, boundary conditions, electromagnetic waves, electromagnetic field

\section{Introduction}

Controllable propagation of electromagnetic wave in a confined space which dimension is smaller than the incident wavelength is an interesting and challenging topic [1]. A new adverse environmental factor has been formed in recent years. This leads to decreasing the efficiency of hardware and staff $[2,3]$. A low-frequency field affects the human especially, because biological functions are largely based on low-frequency rhythms [4]. That is why the creation of different types of screens that improves protection of technical devices, as well as biological effects of magnetic fields is important [5-7]. It is known that multilayered screens material improves the efficiency of screening [8-12]. Depending on the frequency of external magnetic fields, electromagnetic shielding occurs

\footnotetext{
${ }^{1}$ Autor do korespondencji/corresponding author: Viktor Erofeenko, Belarusian State University, 4 Nezavisimosti Avenue, 220050 Minsk, Belarus, (+375)172095248, e-mail: bsu_erofeenko@tut.by

2 Aleksandr Kuts, Belarusian State University, e-mail: sadako1983@mail.ru

${ }^{3}$ Gennady Shushkevich, Belarusian State University, e-mail: g_shu@tut.by
} 
through different physical mechanisms. For static and low-frequency fields, the most important mechanism is flux shunting due to a high magnetic permeability [13]. A number of publications were devoted to the development of an analytical approach to derive simple formulae for the shielding efficiency for different geometries: cubic and cylindrical shields of finite length and spheroidal shields [14]. Sumner et.al. [15] presented a systematic review of a theoretical treatment and comparison with practical realizations. The detail analysis of various shielding geometries and configurations is presented by Rikitake [16].

In the paper the analitycal analysis of electromagnetic waves propagation across the multilayer cylindrical shell is carried out. The aim of the paper is to develop the analitycal method of transformation of boundary conditions to the integral Fredholm equations of the second type using Bessel functions. The relation between the efficiency coefficient $\mathrm{E}$ and a number of layers $\mathrm{n}$ is also analyzed. The developed analitycal method uses average non-local bilateral boundary conditions, which describe the propagation of low-frequency electromagnetic waves across the multilayer cylindrical shell. Average non-local bilateral boundary conditions are related to the waves on either side of the shield. A similar procedure for the electromagnetic field was developed by Erofeenko et.al. [12].

\section{Problem formulation}

The thin-wall cylindrical shell $\mathrm{D}\left\{R_{1}<\rho<R_{2},|z|<\ell, 0 \leq \varphi<2 \pi\right\}$ of the thickness $h=R_{2}-R_{1}$ and the height $2 \ell$ is located in the space $R^{3}$ with the permittivity $\varepsilon_{0}$ and the permeability $\mu_{0}$. Vector $\{\rho, \varphi, z\}$ is cylindrical coordinates at point $\mathrm{O}$ (fig. 1.). The cylinder consists of $n+1$ cylindrical layers $\Omega_{s}\left\{\rho_{s}<\rho<\rho_{s+1},|z|<\ell, 0 \leq \varphi<2 \pi\right\}$ with the permittivity $\mathcal{E}^{(s)}$ and the permeability $\mu^{(\mathrm{s})}, s=0,1, \ldots, n$. Parameters $\Delta_{s}=\rho_{s+1}-\rho_{s}$ and $h=\sum_{s=0}^{n} \Delta_{s}$ are the thickness of the s-layer and thickness of shell $\mathrm{D}$, respectively.

We denote by $\Gamma_{-}\left\{\rho=R_{1},|z|<\ell, 0 \leq \varphi<2 \pi\right\}$ the inner surface and $\Gamma_{+}\left\{\rho=R_{2},|z|<\ell, 0 \leq \varphi<2 \pi\right\}$ the outer surface of the shell D. We introduce the cylindrical surface $\Gamma\left\{\rho=R=0,5\left(R_{1}+R_{2}\right),|z|<\infty, 0 \leq \varphi<2 \pi\right\}$, which divides inner $\mathrm{D}_{1}\{0 \leq \rho<R,|z|<\infty, 0 \leq \varphi<2 \pi\}$ and outer cylindrical region $\mathrm{D}_{2}\{\rho>R,|z|<\infty, 0 \leq \varphi<2 \pi\}$. The surface $\Gamma=\Gamma_{0} \cup \Gamma_{c}$, $\Gamma_{0}\{\rho=R,|z|>\ell, 0 \leq \varphi<2 \pi\}, \quad \Gamma_{c}\{\rho=R,|z|<\ell, 0 \leq \varphi<2 \pi\}$ is the median surface of the shell D. The primary low-frequency magnetic field $\overrightarrow{\mathrm{H}}_{0}=-\mathrm{H}_{0} \overrightarrow{\mathrm{e}}_{x}$ 
influences on the shell $\mathrm{D}$ with a circular frequency $\omega$. The formula for magnetic potential is:

$$
u_{0}=H_{0} \rho \cos \varphi, \quad H_{0}-\text { const. }
$$

Let $u_{1}^{\prime}$ be the magnetic potential of secondary magnetic field at $\mathrm{D}_{1}, u_{2}^{\prime}$ be the magnetic potential of reflected magnetic field at $\mathrm{D}_{2}$ and $u_{j}=u_{0}+u_{j}^{\prime}$ be the total magnetic potential magnetic field at $\mathrm{D}_{j}, j=1,2$. Magnetic fields are defined by the formulas:

$$
\overrightarrow{\mathrm{H}}_{0}=-\operatorname{grad} u_{0}, \quad \overrightarrow{\mathrm{H}}_{j}=-\operatorname{grad} u_{j}, \quad j=1,2
$$

Actual magnetic fields are defined by the formulas:

$$
\overrightarrow{\mathbf{H}}_{0}=-\operatorname{grad} \operatorname{Re}\left(u_{0} \mathrm{e}^{-i \omega t}\right), \quad \overrightarrow{\mathbf{H}}_{j}=-\operatorname{grad} \operatorname{Re}\left(u_{j} \mathrm{e}^{-i \omega t}\right)
$$

where $\omega=2 \pi f$ is the circular frequency of magnetic field.

Fig. 1. Geometry of the problem

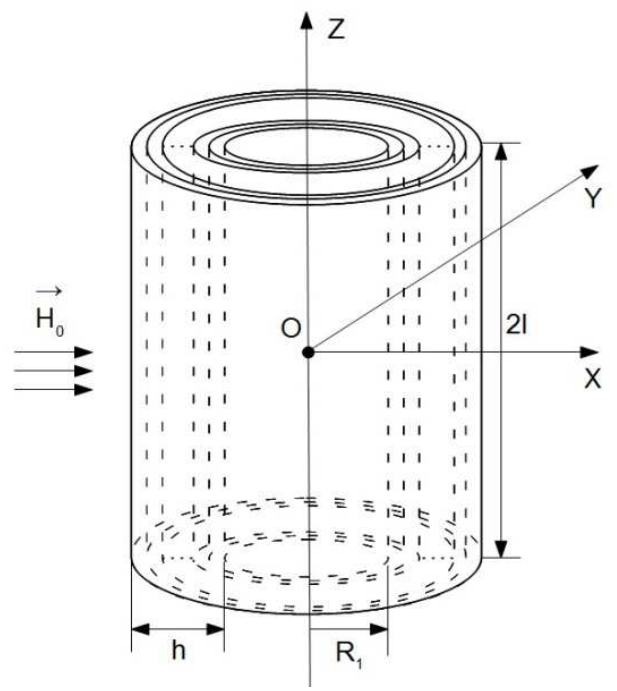

Electromagnetic fields $\overrightarrow{\mathrm{E}}^{(s)}, \overrightarrow{\mathrm{H}}^{(s)}$ satisfy the Maxwell equations in the layers $\Omega_{s}$ of the shell D:

$$
\operatorname{rot} \overrightarrow{\mathrm{E}}^{(s)}=i \omega \mu^{(s)} \overrightarrow{\mathrm{H}}^{(s)}, \quad \operatorname{rot} \overrightarrow{\mathrm{H}}^{(s)}=-i \omega \mu^{(s)} \overrightarrow{\mathrm{E}}^{(s)}, \quad \mu^{(s)}=\mu_{r}^{(s)} \mu_{0}, \quad \varepsilon^{(s)}=\varepsilon_{r}^{(s)} \varepsilon_{0}
$$


Let us formulate the boundary problem of the magnetic field $\overrightarrow{\mathrm{H}}_{0}$ and shell D interaction. We need to define potentials $u_{j}^{\prime} \in C^{2}\left(\mathrm{D}_{j}\right) \cap C^{1}\left(\overline{\mathrm{D}}_{j}\right)$ for the primary potential $u_{0}$ that satisfy Laplace equation:

$$
\Delta u_{j}^{\prime}=0 \text { in } \mathrm{D}_{j}
$$

The boundary conditions take the form:

$$
\begin{aligned}
& \left.u_{2}\right|_{\Gamma_{0}}=\left.u_{1}\right|_{\Gamma_{0}},\left.\frac{\partial u_{2}}{\partial \rho}\right|_{\Gamma_{0}}=\left.\frac{\partial u_{1}}{\partial \rho}\right|_{\Gamma_{0}} \\
& \left.Q_{1} \frac{\partial}{\partial \rho} u_{1}\left(\mathrm{M}_{1}\right)\right|_{\Gamma_{-}}+\left.Q_{2} \frac{\partial}{\partial \rho} u_{2}\left(\mathrm{M}_{2}\right)\right|_{\Gamma_{+}}=F\left(\left.u_{1}\left(\mathrm{M}_{1}\right)\right|_{\Gamma_{-}}-\left.u_{2}\left(\mathrm{M}_{2}\right)\right|_{\Gamma_{+}}\right) \\
& \left.\frac{\partial}{\partial \rho} u_{1}\left(\mathrm{M}_{1}\right)\right|_{\Gamma_{-}}-\left.\frac{\partial}{\partial \rho} u_{2}\left(\mathrm{M}_{2}\right)\right|_{\Gamma_{+}}=F\left(\left.P_{1} u_{1}\left(\mathrm{M}_{1}\right)\right|_{\Gamma_{-}}+\left.P_{2} u_{2}\left(\mathrm{M}_{2}\right)\right|_{\Gamma_{+}}\right)
\end{aligned}
$$

where

$$
\begin{aligned}
& F(u)=(\overrightarrow{\mathrm{n}}, \operatorname{rot}[\overrightarrow{\mathrm{n}}, \operatorname{grad} u])=\frac{\partial^{2} u}{\partial z^{2}}+\frac{1}{\rho^{2}} \frac{\partial^{2} u}{\partial \phi^{2}}, \quad \vec{n}=\overrightarrow{\mathrm{e}}_{\rho} \\
& Q_{j}=i \omega \mu_{0} q_{j}, \quad P_{j}=p_{j} / i \omega \mu_{0}, \quad \mathrm{M}_{j}=\left(\rho=R_{j}, \varphi, z\right), \quad j=1,2,|z|<\ell
\end{aligned}
$$

Finally, the infinity conditions become:

$$
r u_{1}^{\prime}(\mathrm{M}) \rightarrow 0 \text {, when } r \rightarrow \infty, \quad r u_{2}^{\prime}(\mathrm{M}) \rightarrow 0 \text { when } r \rightarrow \infty
$$

where $r$ is the spherical coordinate of point $\mathrm{M}$.

It is noticed that bilateral boundary conditions (8) simulate the magnetic field $\overrightarrow{\mathrm{H}}_{0}$ penetration through D.

\section{Boundary conditions}

The algorithm for calculating the coefficients $p_{j}, q_{j}$ of boundary conditions (8) is as follows. Let us consider transfer matrices for the layers $\Omega_{s}$ : 


$$
\widehat{a}_{s}=\left(\begin{array}{cc}
\cos \left(k_{s} \Delta_{s}\right) & i Z_{s} \sin \left(k_{s} \Delta_{s}\right) \\
i \sin \left(k_{s} \Delta_{s}\right) / Z_{s} & \cos \left(k_{s} \Delta_{s}\right)
\end{array}\right)
$$

where $Z_{s}=\omega \mu_{s} / k_{s}, \quad k_{s}=\omega \sqrt{\varepsilon^{(s)} \mu^{(s)}}, \quad 0 \leq \arg k_{s}<\pi$.

We can use the matrix (12) for the transformation of electromagnetic waves that propagated across the plane layer $\Omega_{s}$ with a thickness $\Delta_{s}$. Let us build the transfer matrix $\widehat{B}$ for the multilayer shell $\mathrm{D}$, where:

$$
\widehat{B}=\widehat{a}_{n} \widehat{a}_{n-1} \ldots \widehat{a}_{1} \widehat{a}_{0}=\left(\begin{array}{ll}
b_{11} & b_{12} \\
b_{21} & b_{22}
\end{array}\right)
$$

Taking into account the matrix elements $b_{j k}$, we can define:

$$
\left.\begin{array}{ll}
p_{1}=\left(b_{22}-1\right) / b_{21}, & p_{2}=\left(b_{11}-1\right) / b_{21} \\
q_{1}=\left(b_{11}-1\right) / b_{12}, & q_{2}=\left(b_{22}-1\right) / b_{12}
\end{array}\right\}
$$

Let us transform non-local boundary conditions (6) and (8). Let us represent magnetic potentials of $\mathrm{D}_{j}$ in the form $u_{j}^{\prime}=v_{j}^{\prime} \cos \varphi, \quad u_{0}=v_{0} \cos \varphi, \quad v_{0}=H_{0} \rho$. We transfer boundary conditions (8) to the median surface $\Gamma_{c}$ for simplify the model. Then we obtain:

$$
\begin{aligned}
& \left.\left(v_{2}^{\prime}-v_{1}^{\prime}\right)\right|_{\Gamma_{0}}=0,\left.\quad\left(\frac{\partial v_{2}^{\prime}}{\partial \rho}-\frac{\partial v_{1}^{\prime}}{\partial \rho}\right)\right|_{\Gamma_{0}}=0 \\
& \left.Q_{1} \frac{\partial}{\partial \rho}\left(v_{1}^{\prime}+v_{0}\right)\right|_{\Gamma_{-}}+\left.Q_{2} \frac{\partial}{\partial \rho}\left(v_{2}^{\prime}+v_{0}\right)\right|_{\Gamma_{+}}= \\
& =\left.\left(\frac{\partial^{2}}{\partial z^{2}}\left(v_{1}^{\prime}+v_{0}\right)-\frac{1}{\rho^{2}} v_{0}\right)\right|_{\Gamma_{-}}-\left.\left(\frac{\partial^{2}}{\partial z^{2}}\left(v_{2}^{\prime}+v_{0}\right)-\frac{1}{\rho^{2}} v_{0}\right)\right|_{\Gamma_{+}}+\left.\frac{1}{\rho^{2}}\left(v_{2}^{\prime}-v_{1}^{\prime}\right)\right|_{\Gamma_{c}} \\
& \left.\left(\frac{\partial v_{1}^{\prime}}{\partial \rho}-\frac{\partial v_{2}}{\partial \rho}\right)\right|_{\Gamma_{c}}=\left.P_{1}\left(\frac{\partial^{2}}{\partial z^{2}}\left(v_{1}^{\prime}+v_{0}\right)-\frac{1}{\rho^{2}}\left(v_{1}^{\prime}+v\right)\right)\right|_{\Gamma_{-}}+ \\
& \quad+\left.P_{2}\left(\frac{\partial^{2}}{\partial z^{2}}\left(v_{2}^{\prime}+v_{0}\right)-\frac{1}{\rho^{2}}\left(v_{2}^{\prime}+v_{0}\right)\right)\right|_{\Gamma_{+}}
\end{aligned}
$$

Let us write the boundary conditions in the compact form: 


$$
\begin{aligned}
& \left.\left(v_{2}^{\prime}-v_{1}^{\prime}\right)\right|_{\Gamma_{0}}=0,\left.\quad\left(\frac{\partial v_{2}^{\prime}}{\partial \rho}-\frac{\partial v_{1}^{\prime}}{\partial \rho}\right)\right|_{\Gamma_{0}}=0 \\
& \left.\left(v_{2}^{\prime}-v_{1}^{\prime}\right)\right|_{\Gamma_{u}}=f_{1}(z)+C_{1},\left.\quad\left(\frac{\partial v_{2}^{\prime}}{\partial \rho}-\frac{\partial v_{1}^{\prime}}{\partial \rho}\right)\right|_{\Gamma_{u}}=f_{2}(z)+C_{2}, \quad|z|<\ell
\end{aligned}
$$

where

$$
\begin{aligned}
& f_{1}(z)=\left.R^{2}\left(Q_{1} \frac{\partial v_{1}^{\prime}}{\partial \rho}-\frac{\partial^{2} v_{1}^{\prime}}{\partial z^{2}}\right)\right|_{\rho=R_{1}}+\left.R^{2}\left(Q_{2} \frac{\partial v_{2}^{\prime}}{\partial \rho}+\frac{\partial^{2} v_{2}^{\prime}}{\partial z^{2}}\right)\right|_{\rho=R_{2}},|z|<l \\
& f_{2}(z)=\left.P_{1}\left(\frac{v_{1}^{\prime}}{R_{1}^{2}}-\frac{\partial^{2} v_{1}^{\prime}}{\partial z^{2}}\right)\right|_{\rho=R_{1}}+\left.P_{2}\left(\frac{v_{2}^{\prime}}{R_{2}^{2}}-\frac{\partial^{2} v_{2}^{\prime}}{\partial z^{2}}\right)\right|_{\rho=R_{2}},|z|<l \\
& C_{1}=\left.R^{2}\left(Q_{2} \frac{\partial v_{0}}{\partial \rho}-\frac{v_{0}}{\rho^{2}}\right)\right|_{\rho=R_{2}}+\left.R^{2}\left(Q_{1} \frac{\partial v_{0}}{\partial \rho}+\frac{1}{\rho^{2}} v_{0}\right)\right|_{\rho=R_{1}}= \\
& =H_{0} R^{2}\left(Q_{1}+Q_{2}+\frac{1}{R_{1} R_{2}}\right) \\
& C_{2}=-\left.P_{1}\left(\frac{\partial^{2} v_{0}}{\partial z^{2}}-\frac{1}{\rho^{2}} v_{0}\right)\right|_{\rho=R_{1}}-\left.P_{2}\left(\frac{\partial^{2} v_{0}}{\partial z^{2}}-\frac{1}{\rho^{2}} v_{0}\right)\right|_{\rho=R_{2}}=H_{0}\left(\frac{P_{1}}{R_{1}}+\frac{P_{2}}{R_{2}}\right)
\end{aligned}
$$

The cylinder $\Gamma_{c}$ of fins $\gamma( \pm)=\{z= \pm \ell, \rho=R, 0 \leq \varphi<2 \pi\}$ must satisfy the requirements of energy limbs around the edges $\mathrm{D}_{\gamma}( \pm)$ :

$$
\int_{\mathrm{D}_{\gamma}( \pm)}|\operatorname{grad} u|^{2} d v<\infty, u(\mathrm{M})=u_{j}(\mathrm{M}), \quad \mathrm{M} \in \mathrm{D}_{j}
$$

This modeling technique is used in [17-20] to calculate the frequency of perfectly thin impermeable screens with ribs. In [12, 21-23] this technique is developed for thin-walled translucent screens (disc, sphere with a hole, ending cylinder) in the case of low-frequency of both electric and magnetic waves. Let consider boundary conditions (18) and (19) as basic boundary conditions for solution of the original problem specified by eqs. (5), (6), (8) and (11). 


\section{Transformation of boundary conditions}

Let us represent unknown functions $v_{j}^{\prime}$ in the integral form:

$$
\begin{aligned}
& v_{1}^{\prime}=\int_{0}^{\infty} x(\lambda) \frac{I_{1}(\lambda \rho)}{I_{1}(\lambda R)} \cos (\lambda z) d \lambda, \quad 0 \leq \rho<R \\
& v_{2}^{\prime}=\int_{0}^{\infty} y(\lambda) \frac{K_{1}(\lambda \rho)}{K_{1}(\lambda R)} \cos (\lambda z) d \lambda, \quad \rho>R
\end{aligned}
$$

where $x(\lambda), y(\lambda)$ are unknown functions, $I_{m}(\lambda R), K_{m}(\lambda R)$ are modified Bessel functions [24].

Substituting integrals (26) into (20), we obtain the integral representation:

$$
f_{j}(z)=\int_{0}^{\infty}\left(x(\lambda) g_{j}(\lambda)+y(\lambda) q_{j}(\lambda)\right) \cos (\lambda z) d \lambda, \quad j=1,2, \quad|z|<\ell
$$

where

$$
\begin{aligned}
& g_{1}(\lambda)=\lambda R^{2}\left(Q_{1} I_{1}^{\prime}\left(\lambda R_{1}\right)+\lambda I_{1}\left(\lambda R_{1}\right)\right) / I_{1}(\lambda R) \\
& q_{1}(\lambda)=\lambda R^{2}\left(Q_{2} K_{1}^{\prime}\left(\lambda R_{2}\right)-\lambda K_{1}\left(\lambda R_{2}\right)\right) / K_{1}(\lambda R) \\
& g_{2}(\lambda)=P_{1}\left(\lambda^{2}+\frac{1}{R_{1}^{2}}\right) \frac{I_{1}\left(\lambda R_{1}\right)}{I_{1}(\lambda R)}, \quad q_{2}(\lambda)=P_{2}\left(\lambda^{2}+\frac{1}{R_{2}^{2}}\right) \frac{K_{1}\left(\lambda R_{2}\right)}{K_{1}(\lambda R)} \\
& K_{1}^{\prime}(x)=-0,5\left(K_{0}(x)+K_{2}(x)\right), \quad I_{1}^{\prime}(x)=0,5\left(I_{0}(x)+I_{2}(x)\right)
\end{aligned}
$$

Let us use the integral transformation:

$$
f(z)=\int_{0}^{\infty} \tilde{f}(\lambda) \cos \lambda z d \lambda, \quad \tilde{f}(\lambda)=\frac{2}{\pi} \int_{0}^{\infty} f(z) \cos \lambda z d z
$$

Let's approximate functions (20) and (21) on interval $0 \leq z<\infty$ :

$$
F_{j}(z)=\left\{\begin{array}{ll}
f_{j}(z), & 0 \leq z<\ell \\
0, & \ell<z<\infty
\end{array}=\int_{0}^{\infty} \tilde{F}_{j}(\lambda) \cos \lambda z d \lambda\right.
$$


Applying the inverse integral transformation of eq. (32) to eq. (33) and considering (27), it gives:

$$
\begin{aligned}
\tilde{F}_{j}(\lambda) & =\frac{2}{\pi} \int_{0}^{\ell} f_{j}(z) \cos \lambda z d z= \\
& =\frac{2}{\pi} \int_{0}^{\infty}\left(x(\eta) g_{j}(\eta)+y(\eta) q_{j}(\eta)\right) \int_{0}^{\ell} \cos \eta z \cos \lambda z d z d \eta= \\
& =\int_{0}^{\infty}\left(x(\eta) g_{j}(\eta)+y(\eta) q_{j}(\eta)\right) L(\lambda, \eta) d \eta
\end{aligned}
$$

where

$$
L(\lambda, \eta)=\left(\frac{\sin (\eta-\lambda) \ell}{\eta-\lambda}+\frac{\sin (\eta+\lambda) \ell}{\eta+\lambda}\right) / \pi
$$

Let $c_{j}(z)$ be piecewise constant function:

$$
c_{j}(z)=\left\{\begin{array}{ll}
c_{j}, & 0 \leq z<\ell \\
0, & \ell<z<\infty
\end{array}=c_{j} \int_{0}^{\infty} q(\lambda) \cos \lambda z d \lambda\right.
$$

where $q(\lambda)=2 \sin (\lambda \ell) /(\pi \lambda)$.

Using functions (33) and (36), we combine boundary conditions (18) and (19) as:

$$
\begin{aligned}
& \left.\left(v_{2}^{\prime}-v_{1}^{\prime}\right)\right|_{\rho=R}=F_{1}(z)+c_{1}(z), \quad 0 \leq z<\infty \\
& \left.\left(\frac{\partial v_{2}^{\prime}}{\partial \rho}-\frac{\partial v_{1}^{\prime}}{\partial \rho}\right)\right|_{\rho=R}=F_{2}(z)+c_{2}(z), \quad 0 \leq z<\infty
\end{aligned}
$$

Let us transform the solution of the problem (eqs. (3), (6), (8), (11)) to the solution of integral Fredholm equations of the second kind, with boundary conditions (38). Functions (26), (32) and (34) occurring in eq. (38) are written in the form of Fourier integrals (32). Applying the inverse integral transform to the eq. (38), we obtain:

$$
y(\lambda)-x(\lambda)=\tilde{F}_{1}(\lambda)+c_{1} q(\lambda)
$$




$$
\lambda\left(y(\lambda) \frac{K_{1}^{\prime}(\lambda R)}{K_{1}(\lambda R)}-x(\lambda) \frac{I_{1}^{\prime}(\lambda R)}{I_{1}(\lambda R)}\right)=\tilde{F}_{2}(\lambda)+c_{2} q(\lambda)
$$

Let us solve set of eqs. (39) and (40) in variables $x(\lambda), y(\lambda)$. It gives:

$$
\left.\begin{array}{l}
x(\lambda)=R\left(\lambda \frac{K_{1}^{\prime}(\lambda R)}{K_{1}(\lambda R)} \tilde{F}_{1}(\lambda)-\tilde{F}_{2}(\lambda)\right)+R q(\lambda)\left(\lambda \frac{K_{1}^{\prime}(\lambda R)}{K_{1}(\lambda R)} C_{1}-C_{2}\right) \\
y(\lambda)=R\left(\lambda \frac{I_{1}^{\prime}(\lambda R)}{I_{1}(\lambda R)} \tilde{F}_{1}(\lambda)-\tilde{F}_{2}(\lambda)\right)+R q(\lambda)\left(\lambda \frac{I_{1}^{\prime}(\lambda R)}{I_{1}^{\prime}(\lambda R)} C_{1}-C_{2}\right)
\end{array}\right\}
$$

Substituting integrals (34) in (41), we obtain set of integral Fredholm equations of the second kind:

$$
\left.\begin{array}{rl}
x(\lambda)= & \left.R \int_{0}^{\infty}\left[x(\eta)\left(\lambda \frac{K_{1}^{\prime}(\lambda R)}{K_{1}(\lambda R)} g_{1}(\eta)-g_{2}(\eta)\right)+y(\eta)\left(\lambda \frac{K_{1}^{\prime}(\lambda R)}{K_{1}(\lambda R)} q_{1}(\eta)-q_{2}(\eta)\right)\right] \times\right] \\
& \times L(\lambda, \eta) d \eta+R q(\lambda)\left(\lambda \frac{K_{1}^{\prime}(\lambda R)}{K_{1}(\lambda R)} C_{1}-C_{2}\right) \\
y(\lambda)= & R \int_{0}^{\infty}\left[x(\eta)\left(\lambda \frac{I_{1}^{\prime}(\lambda R)}{I_{1}(\lambda R)} g_{1}(\eta)-g_{2}(\eta)\right)+y(\eta)\left(\lambda \frac{I_{1}^{\prime}(\lambda R)}{I_{1}(\lambda R)} q_{1}(\eta)-q_{2}(\eta)\right)\right] \times \\
& \times L(\lambda, \eta) d \eta+R q(\lambda)\left(\lambda \frac{I_{1}^{\prime}(\lambda R)}{I_{1}(\lambda R)} C_{1}-C_{2}\right)
\end{array}\right\}
$$

Let us multiply eq. (42) by a factor $\exp (-\lambda h / 4)$. We introduce dimensionless variables of integration $\bar{\lambda}=\lambda R, \bar{\eta}=\eta R$ and unknown functions:

$$
\begin{aligned}
& \bar{x}(\bar{\lambda})=x(\bar{\lambda} / R) \exp (-\bar{\lambda} h / 4 R) / H_{0} R^{2} \\
& \bar{y}(\bar{\lambda})=y(\bar{\lambda} / R) \exp (-\bar{\lambda} h / 4 R) / H_{0} R^{2}
\end{aligned}
$$

The set of dimensionless equations is defined by: 


$$
\left.\begin{array}{l}
\bar{x}(\bar{\lambda})+\int_{0}^{\infty}\left(\bar{K}_{11}(\bar{\lambda}, \bar{\eta}) \bar{x}(\bar{\eta})+\bar{K}_{12}(\bar{\lambda}, \bar{\eta}) \bar{y}(\bar{\eta})\right) d \bar{\eta}=\bar{f}(\bar{\lambda}), 0 \leq \bar{\lambda}<\infty \\
\bar{y}(\bar{\lambda})+\int_{0}^{\infty}\left(\bar{K}_{21}(\bar{\lambda}, \bar{\eta}) \bar{x}(\bar{\eta})+\bar{K}_{22}(\bar{\lambda}, \bar{\eta}) \bar{y}(\bar{\eta})\right) d \bar{\eta}=\bar{g}(\bar{\lambda}), 0 \leq \bar{\lambda}<\infty
\end{array}\right\}
$$

where

$$
\begin{aligned}
& \bar{K}_{11}(\bar{\lambda}, \bar{\eta})=\left(\bar{g}_{2}(\bar{\eta})-\bar{\lambda} \bar{g}_{1}(\bar{\eta}) \frac{K_{1}^{\prime}(\bar{\lambda})}{K_{1}(\bar{\lambda})}\right) \exp ((\bar{\eta}-\bar{\lambda}) \bar{h} / 4) \bar{L}(\bar{\lambda}, \bar{\eta}) \\
& \bar{K}_{12}(\bar{\lambda}, \bar{\eta})=\left(\bar{q}_{2}(\bar{\eta})-\bar{\lambda} \bar{q}_{1}(\bar{\eta}) \frac{K_{1}^{\prime}(\bar{\lambda})}{K_{1}(\bar{\lambda})}\right) \exp ((\bar{\eta}-\bar{\lambda}) \bar{h} / 4) \bar{L}(\bar{\lambda}, \bar{\eta}) \\
& \bar{K}_{21}(\bar{\lambda}, \bar{\eta})=\left(\bar{g}_{2}(\bar{\eta})-\bar{\lambda} \bar{g}_{1}(\bar{\eta}) \frac{I_{1}^{\prime}(\bar{\lambda})}{I_{1}(\bar{\lambda})}\right) \exp ((\bar{\eta}-\bar{\lambda}) \bar{h} / 4) \bar{L}(\bar{\lambda}, \bar{\eta}) \\
& \bar{K}_{22}(\bar{\lambda}, \bar{\eta})=\left(\bar{q}_{2}(\bar{\eta})-\bar{\lambda} \bar{q}_{1}(\bar{\eta}) \frac{I_{1}^{\prime}(\bar{\lambda})}{I_{1}(\bar{\lambda})}\right) \exp ((\bar{\eta}-\bar{\lambda}) \bar{h} / 4) \bar{L}(\bar{\lambda}, \bar{\eta}) \\
& \bar{f}(\bar{\lambda})=\frac{2}{\pi} \sin (\bar{\lambda} \ell)\left(\frac{K_{1}^{\prime}(\bar{\lambda})}{K_{1}(\bar{\lambda})} \bar{C}_{1}-\frac{\bar{C}_{2}}{\bar{\lambda}}\right) \exp (-\bar{\lambda} \bar{h} / 4) \\
& \bar{g}(\bar{\lambda})=\frac{2}{\pi} \sin (\bar{\lambda} \bar{\ell})\left(\frac{I_{1}^{\prime}(\bar{\lambda})}{I_{1}(\bar{\lambda})} \bar{C}_{1}-\frac{\bar{C}_{2}}{\bar{\lambda}}\right) \exp (-\bar{\lambda} \bar{h} / 4) \\
& \bar{g}_{2}(\bar{\lambda})=\left(1+\bar{\lambda}^{2} \bar{R}_{1}^{2}\right) \frac{\bar{P}_{1} I_{1}\left(\bar{\lambda} \bar{R}_{1}\right)}{\bar{R}_{1}^{2} I_{1}(\bar{\lambda})} \\
& \bar{q}_{1}(\bar{\lambda})=\bar{\lambda}\left(\bar{Q}_{1} I_{1}^{\prime}\left(\bar{\lambda} \bar{R}_{1}\right)+\bar{\lambda} I_{1}\left(\bar{\lambda} \bar{R}_{1}\right)\right) / I_{1}(\bar{\lambda}) \\
& \left.K_{1}^{\prime}\left(\bar{\lambda} \bar{R}_{2}\right)-\bar{\lambda} K_{1}\left(\bar{\lambda} \bar{R}_{2}\right)\right) / K_{1}(\bar{\lambda})
\end{aligned}
$$




$$
\begin{aligned}
& \bar{q}_{2}(\bar{\lambda})=\left(1+\bar{\lambda}^{2} \bar{R}_{2}^{2}\right) \frac{\bar{P}_{2} K_{1}\left(\bar{\lambda} \bar{R}_{2}\right)}{\bar{R}_{2}^{2} K_{1}(\bar{\lambda})} \\
& \bar{L}(\bar{\lambda}, \bar{\eta})=\left(\frac{\sin (\bar{\eta}-\bar{\lambda}) \bar{\ell}}{\bar{\eta}-\bar{\lambda}}+\frac{\sin (\bar{\eta}+\bar{\lambda}) \bar{\ell}}{\bar{\eta}+\bar{\lambda}}\right) / \pi
\end{aligned}
$$

where

$$
\begin{aligned}
& \bar{h}=h / R, \quad \bar{\Delta}=\Delta / R, \quad \bar{R}_{j}=R_{j} / R, \quad \bar{\ell}=\ell / R, \quad \bar{Q}_{j}=Q_{j} R, \quad \bar{P}_{j}=P_{j} / R, \\
& \bar{C}_{1}=\bar{Q}_{1}+\bar{Q}_{2}+\bar{\Delta} / \bar{R}_{1} \bar{R}_{2}, \quad \bar{C}_{2}=\bar{P}_{1} / \bar{R}_{1}+\bar{P}_{2} / \bar{R}_{2} .
\end{aligned}
$$

\section{Calculation of a screening coefficient}

Let us calculate the magnetic field at the center of shell $\mathrm{D}$ at the point $\mathrm{O}$. We obtain

$$
\left.\overrightarrow{\mathrm{H}}_{1}\right|_{\substack{\begin{subarray}{c}{\rho=0 \\
\rho=0} }} \\
{ }\end{subarray}}=-\left.\operatorname{grad} u_{1}\right|_{\substack{z=0 \\
\rho=0}}=-H_{0} \overrightarrow{\mathrm{e}}_{x}-\frac{1}{2} \int_{0}^{\infty} \frac{\lambda x(\lambda)}{I_{1}(\lambda R)} d \lambda \overrightarrow{\mathrm{e}}_{x}=-H_{0}(1+L(\bar{x}(\bar{\lambda}))) \overrightarrow{\mathrm{e}}_{x}
$$

where

$$
L(\bar{x}(\bar{\lambda}))=\frac{1}{2} \int_{0}^{\infty} \frac{\bar{\lambda} \bar{x}(\bar{\lambda})}{I_{1}(\bar{\lambda})} \exp (\bar{\lambda} \bar{h} / 4) d \bar{\lambda}
$$

The screening coefficient at the center of cylindrical shell is

$$
K_{e}=\left|\overrightarrow{\mathrm{H}}_{1}\right| /\left|\overrightarrow{\mathrm{H}}_{0}\right|_{\substack{z=0 \\ \rho=0}}=|1+L(\bar{x}(\bar{\lambda}))|
$$

The efficiency coefficient of the shell is defined as

$$
E=1 / K_{e}
$$

Considering the integral on finite the interval of integration from $\mathrm{O}$ to $\mathrm{A}$ and applying the Simpson generalized quadrature formula of fourth-order accuracy to the set of integral Fredholm eq. (44) let's transform the set of integral Fredholm equations into a set of linear algebraic equations [22, 25]: 


$$
\left.\begin{array}{l}
x 0_{n}+\sum_{k=0}^{N} A_{k}\left(K_{n k}^{11} x 0_{k}+K_{n k}^{12} y 0_{k}\right)=f 1_{n} \\
y 0_{n}+\sum_{k=0}^{N} A_{k}\left(K_{n k}^{21} x 0_{k}+K_{n k}^{22} y 0_{k}\right)=g 1_{n}, n=0,1, \ldots, N
\end{array}\right\}
$$

where $\quad K_{n k}^{11}=\bar{K}_{11}\left(\bar{\lambda}_{n}, \bar{\lambda}_{k}\right), \quad K_{n k}^{12}=\bar{K}_{12}\left(\bar{\lambda}_{n}, \bar{\lambda}_{k}\right), \quad K_{n k}^{21}=\bar{K}_{21}\left(\bar{\lambda}_{n}, \bar{\lambda}_{k}\right)$, $K_{n k}^{22}=\bar{K}_{22}\left(\bar{\lambda}_{n}, \bar{\lambda}_{k}\right), \quad f 1_{n}=\bar{f}\left(\bar{\lambda}_{n}\right), \quad g 1_{n}=\bar{g}\left(\bar{\lambda}_{n}\right), \quad \bar{\lambda}_{n}=n h, h=A / N$,

where $N$ is an even number, $A_{k}$ are weighting coefficients calculated by the formulas:

$$
A_{0}=A_{N}=h / 3, \quad A_{2 j}=2 h / 3, \quad A_{2 j+1}=4 h / 3, \quad j=1,2, \ldots, N / 2-1
$$

The solution of the set of eq. (60) $x 0_{n}, y 0_{n}, n=0,1, \ldots, N$, are taken as approximate solution of the set of integral eq. (44) in the points $\bar{\lambda}_{n}$. Calculations showed that to obtain solutions of the set of eq. (60) with accuracy of $10^{-3}$ for considered parameters of the problem we need $A=8, h=0,01$. Input parameters for the computational experiment: $n=2 m$ is a number of layers, where $m=20$ is a number of pairs of layers; $\Delta_{1}=\Delta_{3}=\ldots=\Delta_{n-1}=h_{\text {sum }} / m$ are thicknesses of magnetic layers, where $h_{\text {sum }}=4,8 \cdot 10^{-4} \mathrm{~m}$ is a total thickness of magnetic layer; $\Delta_{2}=\Delta_{4}=\ldots=\Delta_{n}=\alpha \Delta_{1}$ are thicknesses of nonmagnetic layers, where $\alpha=0,125 ; 0,25 ; 0,5 ; \quad \mu_{r}^{(1)}=\mu_{\mathrm{r}}^{(3)}=\ldots=\mu_{r}^{(n-1)}=10^{4}$ are relative permeabilities of magnetic layers; $\varepsilon_{r}^{(1)}=\varepsilon_{r}^{(3)}=\ldots=\varepsilon_{r}^{(n-1)}=i \gamma_{1} / \omega \varepsilon_{0}$ are relative permittivities of magnetic layers, where $\gamma_{1}=10^{7} \mathrm{~S} / \mathrm{m} ; \mu_{r}^{(2)}=\mu_{r}^{(4)}=\ldots=\mu_{r}^{(n)}=1$ are relative permeabilities of nonmagnetic layers; $\varepsilon_{r}^{(2)}=\varepsilon_{r}^{(4)}=\ldots=\varepsilon_{r}^{(n)}=i \gamma_{2} / \omega \varepsilon_{0}$ are relative permittivities of nonmagnetic layers, where $\gamma_{2}=6 \cdot 10^{7} \mathrm{~S} / \mathrm{m} ; \ell=0,5 \mathrm{~m}$ is halflength of the cylinder; $R_{1}=1,1 \cdot 10^{-2} \mathrm{~m}$ is an inner radius of the cylinder; $\Delta_{0}=10^{-3} \mathrm{~m}$ is thickness of nonmagnetic null layer; $\mu_{r}^{(0)}=1$ is relative permeability of nonmagnetic null layer; $\varepsilon_{r}^{(0)}=i \gamma_{2} /\left(\omega \varepsilon_{0}\right)$ is relative permittivity of nonmagnetic null layer. Figures 2 . and 3 . show the relation between the efficiency coefficient $E$ and a number of layers $n$ for some frequencies of magnetic field. 


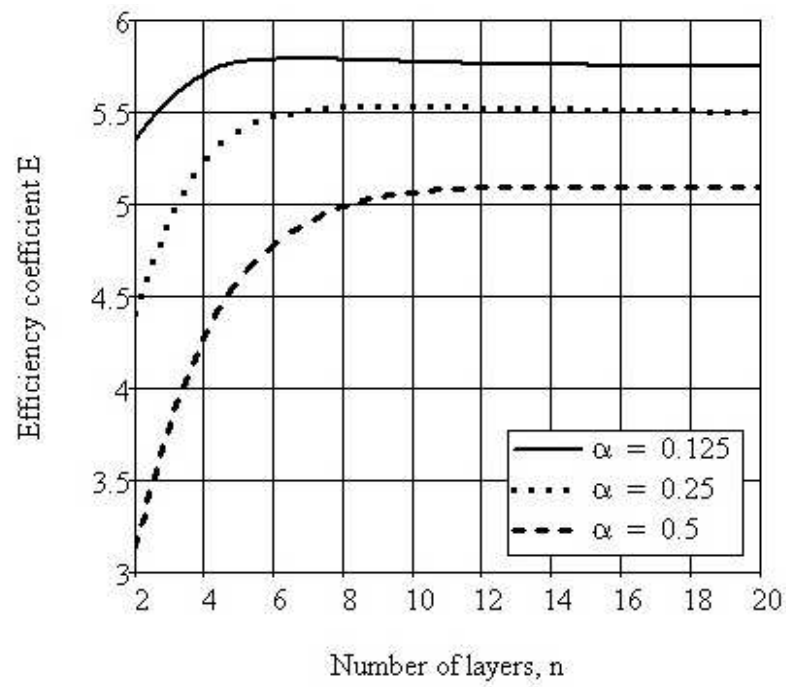

Fig. 2. The relation between the efficiency coefficient $E$ and a number of layers $n, f=200 \mathrm{~Hz}$

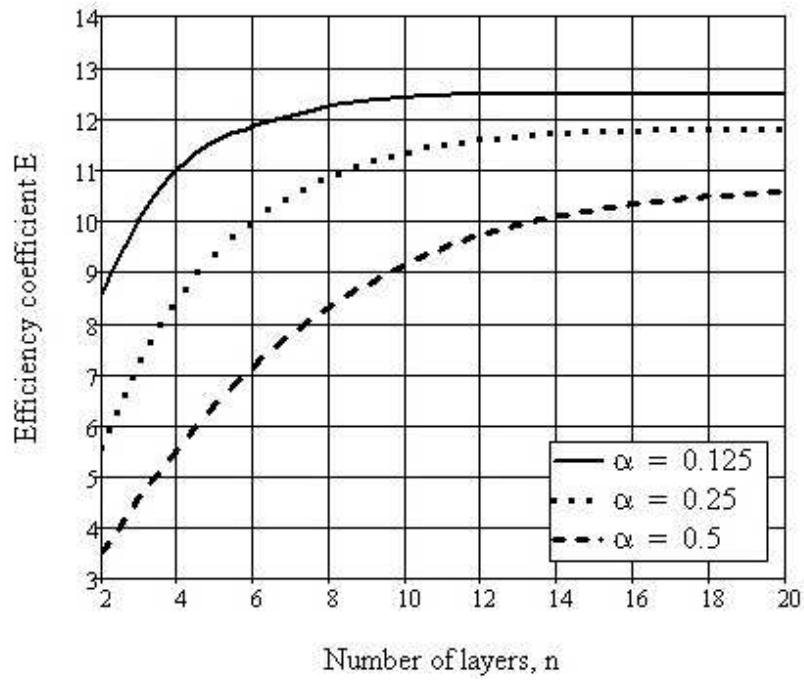

Fig. 3. The relation between the efficiency coefficient $E$ and a number of layers $n, f=500 \mathrm{~Hz}$

\section{Conclusions}

The mathematical model of non-local bilateral boundary conditions of multilayer finite cylindrical shells was built. These non-local bilateral boundary 
conditions describe the propagation of a low-frequency electromagnetic field across layers of the shell. The method of transformation of the boundary conditions to the set of integral Fredholm equations of the second kind was presented. Analytical transformations are based on the Bessel functions. The value of the efficiency coefficient of the shell was also calculated. The results showed that the efficiency coefficient increases with a number of layers for all values of frequencies of magnetic field. The results of mathematic simulation can be used in multilayer shell manufacturing technology.

\section{Acknowledgement}

The research leading to these results has received funding from the People Programme (Marie Curie International Research Staff Exchange) of the European Union's Seventh Framework Programme FP7/2007-2013/ under REA grant agreement No. PIRSES-GA-2013-610547.

\section{References}

[1] Zou S.: Electromagnetic wave propagation in a multilayer silver particle. Chem. Phys. Lett., 454 (2008), 289-293.

[2] Apollonski S.M., Kaliada T.V., Sindalovsky B.E.: A human safety in electromagnetic fields. Politechnika, Petersburg 2012.

[3] Vetoshkin A.G.: Protecting the environment from energy impacts. Vyshaja shkola, Moscow 2010.

[4] Surma S.V., Shchegolev B.F., Vasilieva O.V., Rubanova N.S., Tryrlin V.A.: Weak low-frequency magnetic fields in biology and medicine. Newsletter of the Federal Center of Heart, Blood and Endocrinology, 4(2011), 25-29.

[5] Shapiro D.N.: Electromagnetic Screening. Izd. Dom Intellect, Dolgoprudny 2010.

[6] Sadovnichiy D.N., Markov M.B., Voroncov A.S., Milehin U.M.: Diffraction of electromagnetic pulse on a dielectric cylinder of finite length gradient. J. Tech. Phys., 82(2012), 55-62.

[7] Apollonski A.S., Erofeenko V.T.: Electromagnetic fields in the shielding shells. BSU, Minsk 1988.

[8] Fufayeva L.I., Timofeyev A.B.: The shielding effectiveness of constant magnetic fields by multilayer screens. Works of MAI, 364(1976), 58-63.

[9] Erofeenko V.T: Modeling of processes of penetration of low-frequency magnetic fields through multi-screens. Informatika, 31(2011), 22-32.

[10] Novogorodcev A.B., Petrov V.M.: Pulsed magnetic field shielding double-layered cylindrical screen. Izv. Vuzov. Electromechanika, 5 (1976), 504-508.

[11] Rezinkina M.M.: Parameters of thin electromagnetic shields that provide a decrease in magnetic induction. Tech. Phys., 59(2014), 155-161.

[12] Erofeenko V.T., Shushkevich G.Ch., Grabchikov S.S., Bondarenko V.F.: Model shielding constant magnetic fields multilayer finite cylindrical screen. Informatika, 3(2012), 80-93.

[13] Yashchuk V.V., Lee S.-K., Paperno E.: Magnetic shielding. [In:] D. Budker, D.F.J. Kimball (eds.): Optical magnetometry. Cambridge, London 2013. 
[14] Paperno E., Koide H., Sasada I.: A new estimation of the axial shielding factors for multishell cylindrical shields. J. Appl. Phys., 87(2000), 5959-5691.

[15] Sumner T.J., Pendlebury J. M., Smith K.F.: Convectional magnetic shielding. J. Phys. D: Appl. Phys., 20(1987), 1095-1101.

[16] Rikitake T.: Magnetic and electromagnetic shielding. TERRAPUB and D. Reidel Publ. Co., Tokyo, Dordrecht 1987.

[17] Erofeenko V.T., Kozlovskaja I.S.: Analytical modelling in electrodynamics. BGU, Minsk 2010.

[18] Ufliand Y.S.: The method of dual equations in mathematical physics. Nauka, Leningrad 1977.

[19] Lebedev N.N., Scalskaya I.P.: Application of integral equations to the planar problem of electromagnetic waves on the thin conductive parallel plates. J. Tech Phys., 59 (1989), 3-12.

[20] Shushkevich G.Ch.: Calculation of electrostatic fields by methods of dual, triple equations using addition theorems. GrGU, Grodno 1999.

[21] Erofeenko V.T., Shushkevich G.Ch.: Screening of a low-frequency electric field by a multilayer circular disk. Tech. Phys., 58(2013), 886-871.

[22] Erofeenko V.T., Shushkevich G.Ch.: Screening of low-frequency electric field of thin-walled open spherical shell with the reservoir properties. Electrichestvo, 6(2011), 57-61.

[23] Erofeenko V.T., Kozlovskaya I.S., Shushkevich G.Ch.: Screening of a lowfrequency magnetic field by an open thin-walled spherical shell. Tech. Phys., 55(2010), 1240-1247.

[24] Handbook of Mathematical Functions: with Formulas, Graphs and Mathematical Tables. Eds. M. Abramowitz, I.A. Stegun, Dover, New York 1972.

[25] Fausett L.V.: Numerical Methods Using MathCAD. Prentice Hall, New York 2002.

\section{PRZENIKALNOŚĆ FAL ELEKTROMAGNETYCZNYCH O NISKIEJ CZĘSTOTLIWOŚCI PRZEZ WIELOWARSTWOWA POWLOKE WALCOWA}

\section{Streszczenie}

W artykule przedstawiono wyniki analizy przenikania fal elektromagnetycznych o niskiej częstotliwości przez wielowarstwową powłokę walcową. Zbudowano model matematyczny nielokalnych dwustronnych warunków brzegowych wielowarstwowych powłok walcowych. Te dwustronne nielokalne warunki brzegowe opisują przenikanie pola elektromagnetycznego o niskiej częstotliwości przez warstwy powłoki. Opracowano sposób przekształcania równań brzegowych do całkowych równań Fredholma drugiego rodzaju. Ponadto obliczono wartość współczynnika sprawności powłoki. Do przekształceń analitycznych wykorzystano funkcje Bessela.

Słowa kluczowe: funkcja Bessela, warunki brzegowe, fale elektromagnetyczne, pole elektromagnetyczne

DOI: $10.7862 / \mathrm{rm} .2014 .51$

Otrzymano/received: 20.07.2014 r.

Zaakceptowano/accepted: 22.11.2014 r. 
